The University of Southern Mississippi The Aquila Digital Community

Faculty Publications

Winter 1-1-2019

\title{
Comparing Forward and Backward Chaining In Teaching Olympic Weightlifting
}

James W. Moore

University of Southern Mississippi, james.moore@mycanopy.org

Laura M. Quintero

University of Southern Mississippi

Follow this and additional works at: https://aquila.usm.edu/fac_pubs

Part of the Psychology Commons

\section{Recommended Citation}

Moore, J. W., Quintero, L. M. (2019). Comparing Forward and Backward Chaining In Teaching Olympic Weightlifting. Journal of Applied Behavior Analysis, 52(1), 50-59.

Available at: https://aquila.usm.edu/fac_pubs/16319 


\title{
COMPARING FORWARD AND BACKWARD CHAINING IN TEACHING OLYMPIC WEIGHTLIFTING
}

\author{
James W. Moore and Laura M. Quintero
}

UNIVERSITY OF SOUTHERN MISSISSIPPI

\begin{abstract}
The popularity of Olympic-style weightlifting in fitness routines is growing, but participating in these exercises with improper technique places lifters at increased risk for injury. Fitness training professionals have developed multiple teaching strategies, but have not subjected these strategies to systematic evaluation, particularly with novice lifters. Two strategies recommended by professional training organizations are akin to forward and backward chaining, which have been shown effective at teaching other novel, complex behaviors. The present study compared these forward- and backward-chaining-like strategies to teach novice lifters "the clean" and "the snatch," two Olympic weightlifting movements frequently incorporated into high-intensity training programs. Participants performed lifts taught with forward chaining more accurately than lifts taught with backward chaining.

Key words: behavioral chaining, forward chaining, backward chaining, behavioral skills training, video feedback
\end{abstract}

The clean and the snatch are two complex weightlifting movements that first appeared in the Olympic Games in 1896 as a men's event and was added in 2000 as a women's event (Chiu \& Schilling, 2005). These lifts involve approaching a weighted bar on the ground, and engaging in a forceful, coordinated movement that results in the bar resting either across the shoulders (i.e., the clean) or above the head (i.e., the snatch), followed by a full squat. Although initially popular among competitive weightlifters, these and other Olympic-style lifts have become increasingly common in the fitness plans of novice weightlifters with an estimated $70 \%$ increase over the past 20 years (Burke, Bell, Al-Adawi, Dorvlo, \& Burke, 2014; Keogh, 2009). This increased popularity is likely associated with the increased popularity of high-intensity exercise programs, such as

James W. Moore is now with Canopy Children's Solutions, Jackson, MS. Laura M. Quintero is now with Mississippi State University.

Correspondence concerning this article should be addressed to James W. Moore, Director of Autism Solutions. Canopy Children's Solutions. 1465 Lakeland Drive, Jackson, MS 39216. Contact: james.moore@ mycanopy.org.

doi: $10.1002 /$ jaba. 517
CrossFit ${ }^{\mathrm{TM}}$, which include the clean and snatch as part of their program.

Increased participation in any exercise program offers many health benefits, but weighted movements come with an increased risk of injury relative to alternative forms of exercise (Centers for Disease Control and Prevention, 2015). In a review of six years of injury records from two United States Olympic Training Centers, Calhoon and Fry (1999) found 560 reports of training-related injuries and an estimated 3.3 injuries per 1000 weightlifting hours, with the most common injuries occurring to the back, knees, and shoulders. In a survey of 566 adult CrossFit ${ }^{\mathrm{TM}}$ participants, 31\% reported experiencing an exercise-related injury (Sprey et al., 2016). These injury risks are increased by improper technique (Stone, Fry, Ritchie, Stoesel-Ross, \& Marsit, 1994).

Many novice lifters will recruit training from coaches or personal trainers prior to attempting lifts independently. Although those associated with professional weightlifting have identified proper lifting techniques to minimize injury and maximize exercise gains, there has been no published research on strategies to teach these techniques to novice weightlifters. Instead,

(C) 2018 Society for the Experimental Analysis of Behavior 
most coaches and trainers rely on their personal experience to guide trainees (Takano, 1993). In the absence of systematic evaluation, two general models for training Olympic-style lifts have emerged among trainers (Everett, 2012; Pendlay, 2010; Takano, 1993). Interestingly, both models share tactics familiar to behavior analysts. For instance, both models involve dividing the lifting movements into segments (what behavior analysts would consider a task analysis), teaching the segments in isolation, and then combining individual segments into a chain of responses (similar to what behavior analysts would consider chaining). The first model, often referred to as a "bottom-up" technique, teaches lifts from the floor (i.e., the starting point) and progressively adds segments as they would appear in the lift (similar to forward chaining). The second model, often referred to as a "top-down" technique, teaches lifts from later segments and adds segments in reverse order (similar to backward chaining). The primary distinction between the "topdown" technique and backward chaining is that the former teaches movements within segments in a forward order, but chains segments in backward order.

Behavior analysts have demonstrated the efficacy of both forward and backward chaining in teaching skills such as corsage making (Hur \& Osborne, 1993), operating a washing machine (McDonnell \& McFarland, 1988), tying a shoelace knot (Rayner, 2011), and hitting a baseball (Simek \& O’Brien, 1988). Studies comparing the efficacy of forward and backward chaining have yielded inconsistent results across studies. Weiss (1978) found forward chaining more effcacious in teaching undergraduates to complete contrived tasks. Walls, Zane, and Ellis (1981) found similar outcomes between chaining methods in teaching individuals with mild to moderate intellectual delays to assemble a bicycle brake, a meat grinder, and a carburetor. Slocum and Tiger (2011) found similar efficacy with forward and backward chaining in teaching motor sequences to children. However, these authors have suggested that unique tasks may lend themselves to either forward or backward chaining; Olympic-style weightlifting may be one such task.

Given the growing popularity of Olympiclifting exercise and the increased risk of injury associated with these lifts, a systematic evaluation of teaching techniques is warranted. It is possible that a systematic evaluation of methods used to teach these specific, popular exercises will result in recommendations as to the most efficacious teaching procedure. Use of a more efficacious teaching procedure may result in fewer injuries. Therefore, we compared common coaching techniques that share similarities to forward and backward chaining in teaching two components of Olympic weightlifting. We specifically targeted the clean and the snatch motions with four adult participants.

\section{METHOD}

\section{Participants, Setting, and Materials}

We recruited four participants from a Cross$\mathrm{Fit}^{\mathrm{TM}}$ exercise program that included Olympic lifting as a common training component in the Southern United States. Each participant passed a basic fitness assessment using the Physical Activity Readiness Questionnaire (Adams, 1999) and received an acceptable score on the Functional Movement Screen ${ }^{\mathrm{TM}}$ (Minick et al., 2010); both assessments were administered by the first author, who was certified as a Level One coach by the USA Weightlifting Association and as an Olympic weightlifting coach by CrossFit ${ }^{\mathrm{TM}}$ at the time of the study. All participants were required to receive a physical examination and clearance to participate from their physician.

Patrick was a 58-year-old man in good health who had less than 1 month experience with Olympic weightlifting and reported no prior athletic training. Lee was a 32-year-old woman in good health who had less than 
1 month experience with Olympic weightlifting. Though an avid runner, Lee had no formal athletic training. George was a 45-year-old man with a history of high blood pressure who had 3 months experience with Olympic weightlifting. He played football in high school and college and competed in powerlifting during his 20 s and early 30s. Despite his experience, his lifting form contained critical errors that placed him at risk for injury. Valerie was a 25-year-old woman in good health with less than 1 month experience with Olympic weightlifting. She was a competitive dancer at the time of her participation. As part of the consent process, we asked participants to abstain from weightlifting outside of the study but did not otherwise restrict their participation in the overall exercise program (e.g., running on a treadmill, using weight machines, etc.).

Each participant trained one-on-one with the first author 3 days per week for 2 months, on average, at the gym where the first author was employed as a trainer. Sessions, which lasted $45 \mathrm{~min}$ to $60 \mathrm{~min}$ throughout all phases, took place at one of 10 lifting stations; each station had rubber matted floors and a standard squat rack. Across phases of the study, participants performed clean and snatch lifts, or individual components of these lifts, using different bars to ensure participant safety. During prebaseline practice sessions, participants performed lifts using a polyvinyl chloride (PVC) pipe measuring $1.83 \mathrm{~m}$ in length and weighing $0.45 \mathrm{~kg}$. In accordance with Olympic lifting conventions, this pipe measured $28 \mathrm{~mm}$ in diameter for males and $25 \mathrm{~mm}$ for females. During training sessions, participants used a training bar measuring $1.3 \mathrm{~m}$ in length and $22.2 \mathrm{~mm}$ in diameter, weighing $2.5 \mathrm{~kg}$. These bars had a $10.5-\mathrm{cm}$ loading sleeve (area to add additional weight) and two outer Olympic knurls (markings lifters use for hand placement on the bars). We added weight to bars using standard Olympic lifting bumper plates (rubber coated) weighing 5, 11, 16 , and $20 \mathrm{~kg}$, and smaller iron plates (not rubber coated) weighing 1 and $2.5 \mathrm{~kg}$. Finally, during testing sessions, male participants used a standard 2.13-m long, 28-mm diameter Olympic barbell weighing $20 \mathrm{~kg}$ with a loading sleeve length of $41.28 \mathrm{~cm}$. Female participants used a standard 2.08-m long, 25-mm diameter Olympic barbell weighing $15 \mathrm{~kg}$ with a loading sleeve length of $31.75 \mathrm{~cm}$. Both male and female barbells had two outer knurls and one center knurl. Weight was added to these bars using the same plates described previously.

We video-recorded sessions for data collection using an iPad mini ${ }^{\circledR}$ mounted on a tripod. We scored videos and provided participants with video feedback using Ubersense $($, an application designed specifically for sports video analysis that includes slow-motion playback and drawing tools that allowed us to track the bar path for participants.

\section{Dependent Variables}

We developed a task analysis for both the clean and the snatch (see Supporting Information) based on standards outlined by the United States Weightlifting Association (USAW, 2015). Observers scored videos of each lift in slow motion and coded each step within the task analysis as executed correctly (+) or incorrectly (-). For each lift, we totaled the number of correctly implemented steps, divided by the total number of steps for the lift, and converted the quotient to a percentage.

A second observer scored both cleans and snatches for the purposes of assessing interobserver agreement during 35\%, 39\%, 43\%, and $50 \%$ of sessions for Patrick, Lee, George, and Valerie, respectively. For percentage of correct steps, we compared observer records on an item-by-item basis in the task analysis and scored each item as either an agreement or disagreement. We then totaled the number of steps in agreement, divided by the total number of steps in the lift, and converted the quotient to a percentage. IOA averaged $92 \%, 91 \%$, 
$94 \%$ and $89 \%$ for the clean and $95 \%, 89 \%$, $97 \%$, and $88 \%$ for the snatch for Patrick, Lee, George, and Valerie, respectively.

\section{Procedures}

Pretraining introduction to Olympic lifts. All participants, along with six other nonparticipating trainees, attended a 60-min introductory session on Olympic weightlifting implemented by the first author. This introductory session followed a behavioral skills training model in which we presented didactic instruction via a PowerPoint $($ ) presentation outlining proper technique of each lift, with a video model of both lifts interspersed within the presentation. This $30 \mathrm{~min}$ of didactic instruction was followed by a 30-min practice period in which experimental and nonparticipating trainees rehearsed lifts and received live feedback on the starting mechanics for both lifts. These mechanics included proper placement of the hands (i.e., placing each hand the proper distance from the outer knurls based on the lift), feet (i.e., feet aligned parallel to each other, spaced a shoulder-width apart), and body (i.e., in a squatting position above the bar, with back arched, head up and eyes looking forward). We taught these foundational skills to minimize participants' injury risk during baseline assessments. Participants did not attempt any actual lifts during this introductory session. We required participants to demonstrate mastery-level performance (100\% accuracy) in achieving this starting position across three consecutive opportunities. Positioning errors resulted in feedback including corrective modeling, followed by an immediate opportunity to demonstrate the proper positioning.

Baseline. In baseline sessions, participants performed a minimum of five consecutive cleans and five consecutive snatches with roughly 60 to $90 \mathrm{~s}$ between each lift. We ordered the sequence of cleans and snatches randomly across participants. The total number of baseline cleans and snatches was determined based on visual analysis of the level, trend, and variability for each lift. Male participants used training bars weighted to $20 \mathrm{~kg}$ for cleans and $11 \mathrm{~kg}$ for snatches, and female participants used training bars weighted to $16 \mathrm{~kg}$ for cleans and $6 \mathrm{~kg}$ for snatches. Participants did not receive performance feedback on these lifts.

Training. We divided each lift into four segments and trained these segments as drills. For the clean, these segments were the deadlift, the pull, the power clean, and the squat. For the snatch, these segments were the deadlift, the pull, the power snatch, and the squat. Each of these components included several sub-steps laid out in the Supporting Information. For each participant, we randomly assigned one lift to each of the chaining conditions. We taught Lee and George the clean with forward chaining and the snatch with backward chaining. We taught Patrick and Valerie the snatch with forward chaining and the clean with backward chaining. We initiated training for the lifts simultaneously, to compare forward and backward chaining in an adapted alternating-treatments design. Each training session lasted 45 to $60 \mathrm{~min}$, which included a 10-min warm-up, a 30-min period for drills, and a 5-min period for testing. Participants attended one training session per day, typically 3 days per week. Each participant attended all scheduled training sessions (i.e., there were no cancellations or "no-shows").

During each 30-min period for drills, the trainer began by providing didactic explanation and a live model of the movements targeted prior to participants attempting the drill. Each drill required 10 repetitions of the targeted segment with $30 \mathrm{~s}$ between each repetition. If participants made an error, the trainer provided feedback with a model during the 30 -s period between repetitions. At the completion of the 10 repetitions, the trainer provided additional postlift feedback while reviewing the video of the 10 drill repetitions. After this feedback, participants attempted the full lift from beginning 
to end one time, without feedback. Performance of this final attempt served as our primary dependent variable for that training session. We considered a lift mastered if the participant scored $80 \%$ or greater correct steps for three consecutive trials. ${ }^{1}$

To teach the clean with forward chaining, the order of drills was (a) the deadlift, (b) the pull, (c) the power clean, and (d) the squat. With backward chaining, the order of drills was (a) the squat, (b) the power clean, (c) the pull, and (a) the deadlift. To teach the jerk with forward chaining, the order of drills was (a) the deadlift, (b) the pull, (c) the jerk, and (d) the squat. With backward chaining, the order was (a) the squat, (b) the jerk, (c) the pull, and (a) the deadlift. The drills themselves were identical across forward and backward chaining conditions with the exception that all forward chaining drills began with the bar on the floor (i.e., the natural starting position) and all backward chaining drills began with the bar on a rack that placed the bar at the height necessary to start the targeted movement (e.g., prior to the squat for a clean, the bar must be resting across the front of the shoulders, so the rack presented the bar at shoulder height). The trainer reset the bar on the rack following each drill.

If participants met mastery with one type of lift but not the other, the trainer changed teaching techniques for the nonmastered lift (i.e., changed from backward to forward or vice-versa). This training continued until the participant demonstrated $80 \%$ or greater correct steps on three consecutive trials.

Follow-up. Following mastery for a lift, participants intermittently completed follow-up lifts to assess maintenance using the same weight as training sessions, while they continued participating in training for their

\footnotetext{
${ }^{1}$ There is no current standard for Olympic weightlifting mastery; we set this requirement based upon personal judgment.
}

nonmastered lift. Follow-up lifts occurred after an additional 21 training sessions for Patrick, 2 additional sessions for Lee, 24 sessions for George, and 9 sessions for Valerie. We also conducted follow-up assessments for the subsequently mastered lifts after 20 sessions for Patrick, 2 sessions for Lee, 25 sessions for George, and 9 sessions for Valerie. No feedback was provided for any follow-up trials.

\section{RESULTS}

Figure 1 depicts the percentage of correctly implemented steps during baseline, training, and follow-up for Patrick, Lee, George, and Valerie. Patrick (top panel) performed a mean of $19 \%$ of steps correctly for the snatch and $21 \%$ of steps for the clean during baseline (range, $15 \%$ to $26 \%$ and $15 \%$ to $30 \%$, respectively). For Patrick, the snatch was randomly assigned to the forward-chaining condition and the clean to the backward-chaining condition. He performed $76 \%$ of steps correctly during snatch trials (range, $48 \%$ to $93 \%$ ) and $38 \%$ of steps during clean trials (range, 22\% to 56\%). Training of the snatch via forward chaining led to mastery performance following 11 total training sessions. Following low performance for the clean, we then switched from backward to forward chaining for the clean, which met mastery following 9 additional training sessions. During follow-up trials, Patrick performed 95\% of steps correctly for the snatch (range, $93 \%$ to $96 \%$ ) and $92 \%$ of steps for the clean (range, $89 \%$ to $96 \%$ ).

Lee (second panel) performed 1\% of steps correctly (range, $0 \%$ to $4 \%$ ) for the snatch and $3 \%$ steps correctly (range, $0 \%$ to $4 \%$ ) for the clean during baseline. We randomly assigned the clean to the forward chaining condition and the snatch to the backward chaining condition. She engaged in an average of $64 \%$ of steps correct during clean trials (range, $30 \%$ to $89 \%$ ) and $23 \%$ of steps correct during snatch trials (range, $4 \%$ to $44 \%$ ). Training of the clean via 


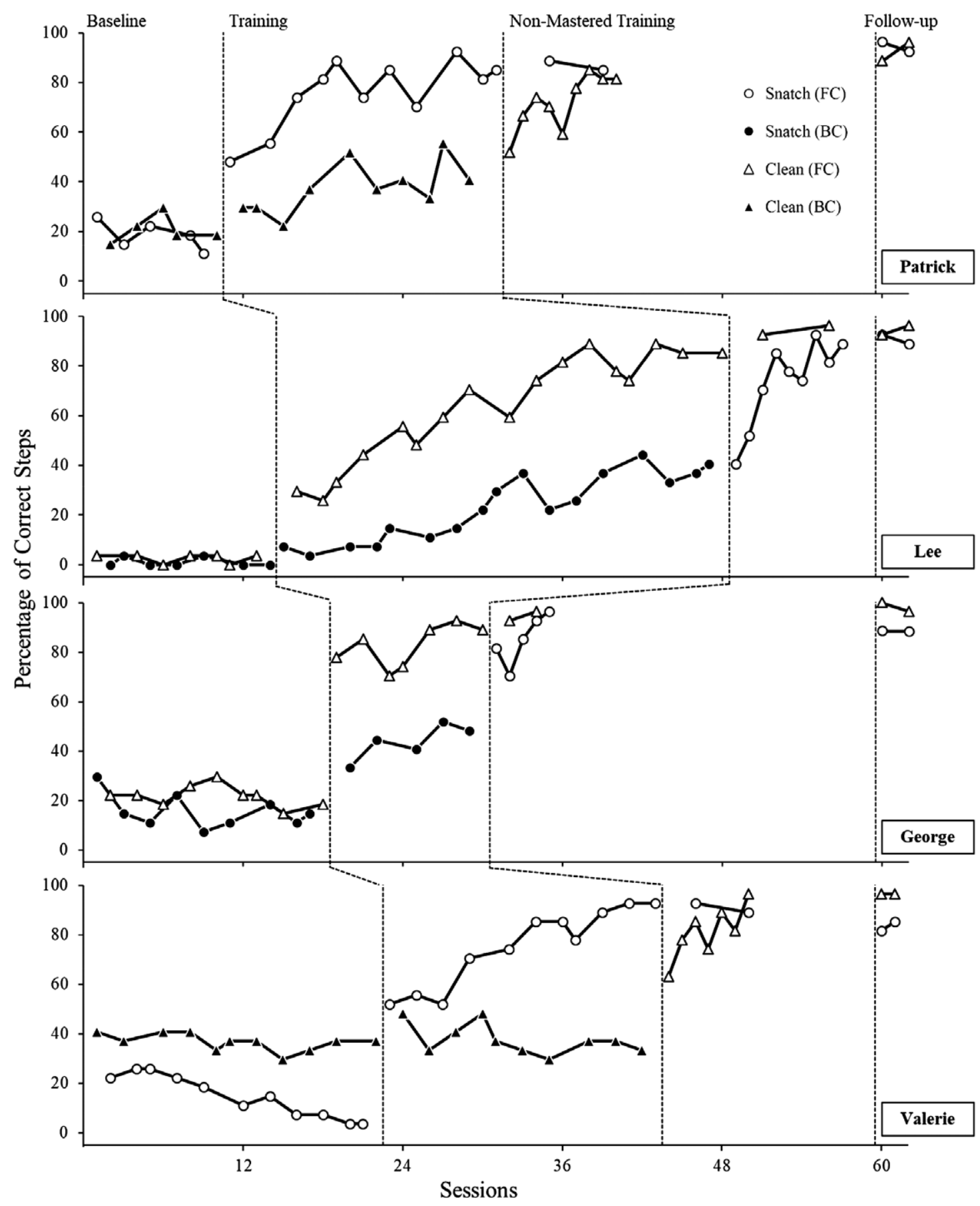

Figure 1. Percentage of correct steps for Patrick (top panel), Lee, (second panel), George (third panel), and Valerie (bottom panel) across Baseline, Training, Nonmastered Training, and Follow-up phases. Open circles represent snatch trials with forward chaining (FC). Closed circles represent snatch trails with backward chaining (BC). Open triangles represent clean trials with FC. Closed triangles represent clean trials with BC. 
forward chaining led to mastery performance following 17 training sessions. Following nonmastery performance for the snatch, we then began training the snatch with forward chaining, with Lee meeting mastery criteria for the snatch within 9 additional training sessions. During follow-up trials, Lee performed $91 \%$ of steps across all trials (range, $89 \%$ to $93 \%$ ) of the snatch and $95 \%$ of steps for the clean (range, $93 \%$ to $96 \%$ ).

George (third panel) performed $16 \%$ and $22 \%$ of steps for the snatch and clean, respectively, during baseline (range, $7 \%$ to $30 \%$ for the snatch and $15 \%$ to $30 \%$ for the clean). We randomly assigned the clean to the forward chaining condition and the snatch assigned to backward chaining. George performed $83 \%$ of steps correctly during clean trials (range, $70 \%$ to $93 \%$ ) and $44 \%$ of steps during snatch trials (range, 33\% to 52\%). Training of the clean via forward chaining led to mastery performance following seven training sessions. Following continued low performance, we then began to train the snatch via forward chaining, which produced mastery after five additional training sessions. During follow-up trials, George performed $96 \%$ of steps for the clean correctly (range, $93 \%$ to $100 \%$ ) and $89 \%$ of steps for the snatch (range, $89 \%$ to $89 \%$ ).

Valerie (bottom panel) performed $15 \%$ of steps correctly for the snatch (range, $4 \%$ to $26 \%$ ) and $37 \%$ of steps for the clean (range, $33 \%$ to $41 \%$, respectively) during baseline. We randomly assigned the snatch to the forwardchaining condition and the clean to the backward-chaining condition. She performed an average of $75 \%$ of steps correctly during snatch trials (range, $52 \%$ to $93 \%$ ) and $38 \%$ of steps during clean trials (range, $30 \%$ to $48 \%$ ) during training. The snatch met mastery performance following 11 forward-chaining sessions. Given continued low performance with the clean, we then began teaching this lift via forward chaining. Following an additional 7 training sessions, the clean met mastery.
During follow-up, Valerie performed $87 \%$ of steps correctly for the snatch (range, $82 \%$ to $93 \%$ ) and $96 \%$ of steps for the clean (range, $96 \%$ to $96 \%)$.

\section{DISCUSSION}

We compared two strategies commonly implemented by coaches and physical trainers, similar to forward and backward chaining, in teaching four novice weight lifters the clean and the snatch. Each of our four participants showed mastery performance following training via forward chaining but showed substantially fewer improvements in performance accuracy when trained via backward chaining. When we changed from backward to forward chaining for those lifts, each participant then reached mastery level performance. Thus, in each comparative application, forward chaining was superior to backward chaining in teaching these Olympic weightlifting movements.

This is the first study of which we are aware that systematically evaluated training Olympicstyle weightlifting. Previous research demonstrated that behavioral teaching can improve athlete performance and safety in sports such as tennis (Allison \& Ayllon, 1980), gymnastics (Wolko, Hrycaiko, \& Martin, 1993), football (Stokes, Luiselli, Reed, \& Fleming, 2010), track (Shapiro \& Shapiro, 1985), and swimming (Hazen, Johnstone, Martin, \& Srikameswaran, 1990), but the application of these procedures to weightlifting is novel. As novices increasingly incorporate these "explosive movement" lifts into their training programs, additional research should be directed towards minimizing injury risk in this burgeoning sport.

Our results also contribute to the literature comparing forward and backward chaining (e.g., Hur \& Osborne, 1993; McDonnell \& McFarland, 1988; Rayner, 2011; Simek \& O'Brien, 1988), which have yielded inconsistent results when comparing the efficacy of 
forward and backward chaining. Some response chains may, however, more readily lend themselves to either forward or backward chaining (Slocum \& Tiger, 2011). In Slocum and Tiger, both forward and backward chaining resulted in participants mastering motor sequences that consisted of discrete movements in which, perhaps importantly, it was possible for a participant to engage in a correct movement later in the chain following an incorrect movement earlier in the chain. For instance, in a sequence of touch your head, touch your nose, and raise your arms, it is physically possible to raise one's hands without first touching one's nose. However, in movements such as the clean, it may not be possible to correct for an error in the chain (e.g., if the bar is not lifted to the hips before initiating the final pull under the bar). Backward chaining did not produce mastery performance for any participant on any lift. It is possible that (a) forward chaining ensured mastery performance of all initial movements before moving to later movements, (b) we were unable to appropriately simulate the position attained during these initial movements with the starting locations for backward chaining, (c) the initial movements produced physical momentum to aide in lift completion during forward chaining relative to backward chaining, and/or (d) initial movements generate visual and kinesthetic discriminative stimuli that occasion subsequent responses that are not present during backward chaining. Future research may compare forward and backward chaining across similar complex movements, such as operating heavy equipment, striking a soccer ball with the head, or completing a tackle.

We offer some important caveats to this discussion in that our procedures differed from traditional chaining procedures in the following ways. First, across both procedures, we taught segments collectively rather than individual steps. Chaining, in which the task is broken into individual steps, is often contrasted with whole-task training, in which the task is taught in its entirety. If we consider those procedures to represent a spectrum, rather than dichotomous options for training, our focus on training segments could be viewed as a procedural middle ground. Our backward chaining procedure was also unique, which may have accounted for its decreased efficacy. Typically, backward chaining involves sequentially linking individual steps from the end of the chain to the beginning. Instead, our backward chaining involved linking "segments" of lifts together. Although these procedures diverged from common chaining procedures, they represented the techniques commonly used by trainers and coaches for teaching these lifts. Future evaluations could compare more traditional chaining to our model to determine if these modifications yielded improved or worsened outcomes.

One of our more surprising outcomes was that we did not see generalization across lifts, most notably from forward chaining to backward chaining conditions. Regardless of the assigned teaching condition, the first two segments of the clean and the jerk were identical. Our comparison may have inadvertently favored backward chaining in that mastering the first two segments in one lift via forward chaining could produce mastery of those same skills in the lift targeted via backward chaining, but the final two movements of each lift differed. Thus, it would be less likely for generalization of skills to occur from the backward chaining condition to the forward chaining condition. Despite this, we still observed limited acquisition in each lift during the backward chaining teaching procedure.

The two participants with the most athletic experience demonstrated the most rapid lift acquisition. George, a high school and college football player, met mastery for the clean in 7 trials and the snatch in 5 trials when exposed to forward chaining. Similarly, Valerie, a competitive dancer, required 11 trials to reach mastery for the snatch and 7 trials for the clean. Prior athletic experience may impact the 
efficiency of training procedures and, as such, may influence a trainer's use of these procedures across trainees as a function of a history or no history with past athletic activities. Future research should evaluate prior athletic experience as a predictive factor for training efficiency.

We would also highlight that our initial baseline conditions included instructions, modeling, and rehearsal with video feedback while targeting the total task (lift). Boyer, Miltenberger, Batsche, and Fogel (2009) found that providing video modeling and video feedback was sufficient to achieve mastery performance in gymnastics, but these procedures produced less than $50 \%$ accuracy across our participants. We believe these results highlight the importance of breaking such complex movements into component segments, particularly for novice lifters, who are at increased risk for injury.

We presented our data in an aggregate form, but future researchers may evaluate performance of individual component skills for each lift to identify patterns exhibited on a step-bystep basis across participants. Some steps may rarely occasion errors and can be deemphasized during training or combined with other steps to increase training efficiency, whereas other steps may occasion frequent errors that can be emphasized or further broken into components during training drills. It may also be possible to identify critical steps where errors prevent further accurate performance of a lift but that, once corrected, allow performance improvement in subsequent steps without direct training.

Our study is the first attempt to create a formal and detailed task analysis of the clean and the snatch. This task analysis should be validated by additional experts and their trainees. We are currently recruiting such experts to evaluate the task analysis and to offer pre- and posttraining ratings of new participants to assess the more general efficacy and social validity of our procedures.

\section{REFERENCES}

Adams, R. (1999). Revised physical readiness questionnaire. Canadian Family Physician, 45, 992-1005.

Allison, M. G., \& Ayllon, T. (1980). Behavioral coaching in the development of skills in football, gymnastics, and tennis. Journal of Applied Behavior Analysis, 13, 297-314. https://doi.org/10.1901/jaba.1980.13-297.

Boyer, E., Miltenberger, R. G., Batsche, C., \& Fogel, V. (2009). Video modelling by experts with video feedback to enhance gymnastics skills. Journal of Applied Behavior Analysis, 42, 855-860. https://doi.org/10. 1901/jaba.2009.42-855.

Burke, D. T., Bell, R., Al-Adawi, S., Dorvlo, A., \& Burke, D. P. (2014). Rate of injury and subjective benefits of gravitational wellness weightlifting. Journal of Sports Medicine, 5, 215-221. https://doi.org/10. 2147/OAJSM.S64078.

Calhoon, G., \& Fry, A. C. (1999). Injury rates and profiles of elite competitive weightlifters. Journal of Athletic Training, 34, 232. https://www.ncbi.nlm.nih. gov/pubmed/16558570.

Centers for Disease Control (2015). Physical activity and health. Retrieved from https://www.cdc.gov/ physicalactivity/basics/pa-health/index.htm.

Chiu, L. Z. F, \& Schilling, B. K. (2005). A primer on weightlifting: From sport to sport training. Strength and Conditioning Journal, 27, 42-48. https://journals. lww.com/nsca scj/Fulltext/2005/02000/A_Primer_ on_Weightlifting_From_Sport_to_Sports.8.aspx.

Everett, G. (2012). Olympic weightlifting: A complete guide for athletes and coaches ( $3^{\text {rd }} \mathrm{Ed}$.). San Diego, CA: Catalyst Athletics, LLC.

Hazen, A., Johnstone, C., Martin, G. L., \& Srikameswaran, S. (1990). A videotaping feedback package for improving skills of youth competitive swimmers. The Sport Psychologist, 4, 213-227. https:// doi.org/10.1123/tsp.4.3.213.

Hur, J., \& Osborne, S. (1993). A comparison of forward and backward chaining methods used in teaching corsage making skills to mentally retarded adults. The British Journal of Developmental Disabilities, 38, 108117. https://doi.org/10.1179/bjdd.1993.013.

Keogh, J. W. L. (2009). Weightlifting. In D. J. Caine, P. A. Harmer, \& M. A. Schiff (Eds), Epidemiology of injury in Olympic sports (pp. 336-350). Oxford, UK: Wiley-Blackwell. 9781444316872.ch24

McDonnell, J., \& McFarland, S. (1988). A comparison of forward and concurrent chaining strategies in teaching laundromat skills to students with severe handicaps. Research in Developmental Disabilities, 9, 177$194 . \quad$ https://doi.org/10.1016/0891-4222(88) 90051-0.

Minick, K. I., Kiesel, K.B., Burton, L., Taylor, A., Plisky, P., \& Butler, R.J. (2010). Interrater reliability of the Functional Movement Screen. Journal of 
Strength and Conditioning Research, 24, 479-486. https://doi.org/10.1519/JSC.0b013e3181c09c04.

Pendlay, G. (2010). Olympic weightlifting techniques [Video lecture]. Available from https://www. otpbooks.com/product/glenn-pendlay-weightliftingvideo/?ref=20.

Rayner, C. (2011). Teaching students with autism to tie a shoelace knot using video prompting and backward chaining. Developmental Neurorehabilitation, 6, 339347. https://doi.org/10.3109/17518423.2011.606508.

Shapiro, E. S., \& Shapiro, S. (1985). Behavioral coaching in the development of skills in track. Behavior Modification, 9, 211-224. https://doi.org/10. $1177 / 01454455850092005$.

Simek, T. C., \& O’Brien, R. M. (1988). A chaining-mastery, discrimination training program to teach Little Leaguers to hit a baseball. Human Performance, 1, 73-84. https://doi.org/10.1207/s15327043hup0101_4.

Slocum, S. K., \& Tiger, J. H. (2011). An assessment of the efficiency of and child preference for forward and backward chaining. Journal of Applied Behavior Analysis, 44, 793-805. http://doi.org/10.1901/jaba. 2011.44-793.

Sprey, J. W. C., Ferreira, T., de Lima, M. V., Duarte, A., Jorge, P. B., \& Santilli, C. (2016). An epidemiological profile of CrossFit athletes in Brazil. Orthopaedic Journal of Sports Medicine, 4, 1-8. https://doi.org/10. $1177 / 232596711666706$.

Stokes, J. V., Luiselli, J. K., Reed, D. D., \& Fleming, R. K. (2010). Behavioral coaching to improve offensive line pass-blocking skills of high school football athletes. Journal of Applied Behavior Analysis, 43, 463-472. https://doi.org/10.1901/jaba. 2010.43-463.

Stone, M. H., Fry, A. C., Ritchie, M., Stoessel-Ross, L., \& Marsit, J. L. (1994). Injury potential and safety aspects of weightlifting movements. Strength and Conditioning
Journal, 16, 15-21. https://journals.lww.com/nsca-scj/ Citation/1994/06000/Injury_Potential_and_Safety_ Aspects_of.2.aspx.

Takano, B. (1993). Classic coaching techniques: Coach optimal technique in the snatch and clean and jerk Part 1. National Strength and Conditioning Association Journal, 15, 33-39.

United States Weightlifting Association (2015). Weightlifting and sports performance coaching course manual [Data file]. Retrieved from http://www.teamusa.org/ USA Weightlifting/Weightlifting101/

Walls, R. T., Zane, T., \& Ellis, W. D. (1981). Forward and backward chaining and whole task methods. Behavior Modification, 5, 61-74. https://doi.org/ 10-1177/014544558151005.

Weiss, K. M. (1978). A comparison of forward and backward procedures for the acquisition of response chains in humans. Journal of the Experimental Analysis of Behavior, 29, 255-259. https://doi.org./10.1901/ jeab.1978.29-255.

Wolko, K. L., Hrycaiko, D. W., \& Martin, G. L. (1993). A comparison of two self-management packages to standard coaching for improving practice performance of gymnasts. Behavior Modification, 17, 209-223. https.//doi.org/10.1177/01454455930172007.

Received March 16, 2017

Final Acceptance March 29, 2018

Action Editor, Jeffrey Tiger

\section{SUPPORTING INFORMATION}

Additional Supporting Information may be found in the online version of this article at the publisher's website. 